\title{
MOMENTS OF LOWER GENERALIZED ORDER STATISTICS FROM DOUBLY TRUNCATED CONTINUOUS DISTRIBUTIONS AND CHARACTERIZATIONS
}

\author{
Devendra Kumar*
}

\begin{abstract}
In this paper, we derive recurrence relations for moments of lower generalized order statistics within a class of doubly truncated distributions. Inverse Weibull, exponentiated Weibull, power function, exponentiated Pareto, exponentiated gamma, generalized exponential, exponentiated log-logistic, generalized inverse Weibull, extended type I generalized logistic, logistic and Gumble distributions are given as illustrative examples. Further, recurrence relations for moments of order statistics and lower record values are obtained as special cases of the lower generalized order statistics, also two theorems for characterizing the general form of distribution based on single moments of lower generalized order statistics are given.
\end{abstract}

\section{Introduction}

Kamps [11] introduced the concept of generalized order statistics (gos). It is know that ordinary order statistics, upper record values and sequential order statistics are special cases of gos. In this paper we will consider the lower generalized order statistics (lgos). It can be shown that order statistics, lower record values are special cases of lgos.

Let $n \in N, k \geq 1, m \in \Re$, be the parameters such that

$$
\gamma_{r}=k+(n-r)(m+1)>0,
$$

for all $1 \leq r \leq n$. Then $X^{*}(1, n, m, k), \cdots, X^{*}(n, n, m, k)$ are $n$ lgos from an absolutely continuous distribution function $(d f) F(x)$ with the

Received April 28, 2012; Accepted May 23, 2013.

2010 Mathematics Subject Classification: Primary 62G30, 62E10.

Key words and phrases: lower generalized order statistics, order statistics, lower record values, recurrence relations and characterization. 
corresponding probability density function $(p d f) f(x)$. Their joint $p d f$ is

$$
k\left(\prod_{j=1}^{n-1} \gamma_{j}\right)\left(\prod_{i=1}^{n-1}\left[F\left(x_{i}\right)\right]^{m_{i}} f\left(x_{i}\right)\right)\left[F\left(x_{n}\right)\right]^{k-1} f\left(x_{n}\right)
$$

on the cone

$$
F^{-1}(1)>x_{1} \geq x_{2} \geq \ldots \geq x_{n}>F^{-1}(0) .
$$

For simplicity we shall assume $m_{1}=m_{2}=\cdots=m_{n-1}=m$.

The marginal $p d f$ of the $r$-th $\operatorname{lgos}, X^{*}(r, n, m, k)$ is

$$
f_{X^{*}(r, n, m, k)}(x)=\frac{C_{r-1}}{(r-1) !}[F(x)]^{\gamma_{r}-1} f(x) g_{m}^{r-1}(F(x))
$$

and the joint $p d f$ of $X^{*}(r, n, m, k)$ and $X^{*}(s, n, m, k), 1 \leq r<s \leq n$ is expressed from (1.1) as

$$
\begin{array}{r}
f_{X^{*}(r, n, m, k), X^{*}(s, n, m, k)}(x, y)=\frac{C_{s-1}}{(r-1) !(s-r-1) !}[F(x)]^{m} f(x) g_{m}^{r-1}(F(x)) \\
\times\left[h_{m}(F(y))-h_{m}(F(x))\right]^{s-r-1}[F(y)]^{\gamma_{s}-1} f(y), x>y,
\end{array}
$$

where

$$
\begin{aligned}
& C_{r-1}=\prod_{i=1}^{r} \gamma_{i}, \gamma_{i}=k+(n-i)(m+1), \\
& h_{m}(x)=\left\{\begin{array}{l}
-\frac{1}{m+1} x^{m+1}, m \neq-1 \\
-\ln x, m=-1
\end{array}\right.
\end{aligned}
$$

and

$$
g_{m}(x)=h_{m}(x)-h_{m}(1), x \in[0,1) .
$$

We shall also take $X^{*}(0, n, m, k)=0$. If $m=0, k=1$, then $X^{*}(r, n, m, k)$ reduced to the $(n-r+1)$-th order statistics, $X_{n-r+1: n}$ from the sample $X_{1}, X_{2}, \ldots, X_{n}$ and when $m=-1$, then $X^{*}(r, n, m, k)$ reduced to the $r-$ th lower $k$ record value [Pawlas and Szynal, [17]]. The work of Burkschat et al. [5] may also refer for lower generalized order statistics. Recurrence relations are interesting in their own right. They are useful in reducing the number of operations necessary to obtain a general form for the function under consideration. Furthermore, they are used in characterizing distributions, which in important area, permitting the identification of population distribution from the properties of the sample. 
Many recurrence relations between moments of generalized order statistics are available in the literature. Reference may be made to Cramer and Kamps [6], Pawlas and Szynal [17], Ahmad and Fawzy [4], Ahmad [2], Khan et al. [16] and references therein.

Characterizations of particular distributions based on the moments and conditional moments of order statistics were presented by some authors such as Wu and Ouyang [18], Grudzien and Szynal [9], Khan and Abouammoh [14], Ahmad [1], Govindarajulu [8], amongs others.

Characterizations based on gos have been studied by some authors. Keseling [13] characterized some continuous distributions based on conditional distributions of gos. Bieniek and Szynal [4] characterized some distributions via linearity of regression of gos. Cramer et al. [7] gave a unifying approach on characterization via linear regression of ordered random variables. Khan et al. [15] characterized some continuous distributions through conditional expectation of functions of gos.

Kamps [12] investigated the importance of recurrence relations of order statistics in characterization.

The doubly truncated case of a distribution is the most general case since it includes the right, left and non-truncated distribution as special cases.

Now if for given $P_{1}$ and $Q_{1}$

$$
\int_{-\infty}^{Q_{1}} f_{1}(x) d x=Q \text { and } \int_{-\infty}^{P_{1}} f_{1}(x) d x=P,
$$

where $f_{1}(x)$ is the $p d f$ of $X$. Then the truncated $p d f$ is given by

$$
f(x)=\frac{f_{1}(x)}{P-Q}, x \in\left(Q_{1}, P_{1}\right)
$$

and the corresponding $d f$ by

$$
F(x)=\frac{1}{P-Q}\left[F_{1}(x)-Q\right], x \in\left(Q_{1}, P_{1}\right) .
$$

Suppose the distribution function is of the following general form

$$
F_{1}(x)=e^{-a / h(x)}, x \in(\alpha, \beta),
$$

where $a \neq 0$ is a constant and $h(x)$ is continuous, monotonic and differentiable function of $x$ in the interval $[\alpha, \beta]$. Then truncated $p d f f(x)$ is given by

$$
f(x)=\frac{a}{(P-Q) h^{2}(x)} e^{-a / h(x)} h^{\prime}(x), x \in\left(Q_{1}, P_{1}\right)
$$


and the corresponding truncated $p d f f(x)$ by

$$
F(x)=-Q_{2}+\frac{h^{2}(x)}{a h^{\prime}(x)} f(x),
$$

where

$$
Q_{2}=\frac{Q}{P-Q}
$$

\section{Recurrence relations for single moments}

Theorem 2.1. For the distribution given in (1.7), $n \in N, 2 \leq r \leq$ $n, k \geq 1$, and $m>-1$

$$
\begin{aligned}
& E\left[X^{* j}(r, n, m, k)\right]-E\left[X^{* j}(r-1, n, m, k)\right] \\
&=\frac{j}{a \gamma_{r}}\left\{Q E\left[\phi\left(X^{*}(r, n, m, k)\right) e^{a / h\left(X^{*}(r, n, m, k)\right)}\right]-E\left[\phi\left(X^{*}(r, n, m, k)\right)\right]\right\}, \\
& m \neq-1
\end{aligned}
$$

and for $m=-1$

$$
\begin{aligned}
& E\left[X^{* j}(r, n,-1, k)\right]-E\left[X^{* j}(r-1, n,-1, k)\right] \\
& =\frac{j}{a k}\left\{Q E\left[\phi\left(X^{*}(r, n,-1, k)\right) e^{a / h\left(X^{*}(r, n,-1, k)\right)}\right]-E\left[\phi\left(X^{*}(r, n,-1, k)\right)\right]\right\} .
\end{aligned}
$$

where

$$
\phi(x)=\frac{x^{j-1} h^{2}(x)}{h^{\prime}(x)} .
$$

Proof. We have from (1.2)

$$
E\left[X^{* j}(r, n, m, k)\right]=\frac{C_{r-1}}{(r-1) !} \int_{Q_{1}}^{P_{1}} x^{j}[F(x)]^{\gamma_{r}-1} f(x) g_{m}^{r-1}(F(x)) d x .
$$

Integrating by parts treating $[F(x)]^{\gamma_{r}-1} f(x)$ for integration and rest of the integrand for differentiation, we get

$$
\begin{aligned}
& E\left[X^{* j}(r, n, m, k)\right] \\
& =E\left[X^{* j}(r-1, n, m, k)\right]-\frac{j C_{r-1}}{\gamma_{r}(r-1) !} \int_{Q_{1}}^{P_{1}} x^{j-1}[F(x)]^{\gamma_{r}} g_{m}^{r-1}(F(x)) d x
\end{aligned}
$$


the constant of integration vanishes since the integral considered in (2.3) is a definite integral. On using (1.7), we obtain when $m>-1$ that

$$
\begin{aligned}
& E\left[X^{* j}(r, n, m, k)\right]=E\left[X^{* j}(r-1, n, m, k)\right] \\
& -\frac{j C_{r-1}}{\gamma_{r}(r-1) !} \int_{Q_{1}}^{P_{1}} x^{j-1}[F(x)]^{\gamma_{r}-1}\left\{-Q_{2}+\frac{h^{2}(x) f(x)}{a h^{\prime}(x)}\right\} g_{m}^{r-1}(F(x)) d x
\end{aligned}
$$

which can be written as

$$
\begin{aligned}
& E\left[X^{* j}(r, n, m, k)\right]-E\left[X^{* j}(r-1, n, m, k)\right] \\
& =\frac{j Q_{2} C_{r-1}}{\gamma_{r}(r-1) !} \int_{Q_{1}}^{P_{1}} x^{j-1}[F(x)]^{\gamma_{r}-1} g_{m}^{r-1}(F(x)) d x \\
& \quad-\frac{j C_{r-1}}{a \gamma_{r}(r-1) !} \int_{Q_{1}}^{P_{1}} \frac{x^{j-1} h^{2}(x)}{h^{\prime}(x)}[F(x)]^{\gamma_{r}-1} f(x) g_{m}^{r-1}(F(x)) d x \\
& =\frac{j Q C_{r-1}}{a \gamma_{r}(r-1) !} \int_{Q_{1}}^{P_{1}} \frac{x^{j-1} h^{2}(x) e^{a / h(x)}}{h^{\prime}(x)}[F(x)]^{\gamma_{r}-1} f(x) g_{m}^{r-1}(F(x)) d x \\
& \quad-\frac{j C_{r-1}}{a \gamma_{r}(r-1) !} \int_{Q_{1}}^{P_{1}} \frac{x^{j-1} h^{2}(x)}{h^{\prime}(x)}[F(x)]^{\gamma_{r}-1} f(x) g_{m}^{r-1}(F(x)) d x
\end{aligned}
$$

and hence the result given in (2.1).

When $m=-1$,

$$
\begin{aligned}
& E\left[X^{* j}(r, n,-1, k)\right]-E\left[X^{* j}(r-1, n,-1, k)\right] \\
& =-\frac{j k^{r-1}}{(r-1) !} \int_{Q_{1}}^{P_{1}} x^{j-1}[F(x)]^{k-1}\left\{-Q_{2}+\frac{h^{2}(x) f(x)}{a h^{\prime}(x)}\right\} g_{-1}^{r-1}(F(x)) d x \\
& =\frac{j Q_{2} k^{r-1}}{(r-1) !} \int_{Q_{1}}^{P_{1}} x^{j-1}[F(x)]^{k-1} g_{-1}^{r-1}(F(x)) d x \\
& \quad-\frac{j k^{r-1}}{a(r-1) !} \int_{Q_{1}}^{P_{1}} \frac{x^{j-1} h^{2}(x)}{h^{\prime}(x)}[F(x)]^{k-1} f(x) g_{-1}^{r-1}(F(x)) d x
\end{aligned}
$$

rewriting the above equation we get the result given in (2.2).

\section{Special cases}

i) Putting $m=0, k=1$ in (2.1), we can get the recurrence relations for single moment of order statistics as 


$$
\begin{aligned}
& E\left(X_{n-r+1: n}^{j}\right)=E\left(X_{n-r+2: n}^{j}\right) \\
& \quad+\frac{j}{a(n-r+1)}\left\{Q E\left(\phi\left(X_{n-r+1: n}\right) e^{a / h\left(x_{n-r+1: n}\right)}\right)-E\left(\phi\left(X_{n-r+1: n}\right)\right)\right\} .
\end{aligned}
$$

That is

$$
\begin{aligned}
E\left(X_{r: n}^{j}\right)=E & \left(X_{r-1: n}^{j}\right) \\
& -\frac{j}{a(r-1)}\left\{Q E\left(\phi\left(X_{r-1: n}\right) e^{a / h\left(x_{r-1: n}\right)}\right)-E\left(\phi\left(X_{r-1: n}\right)\right)\right\} .
\end{aligned}
$$

ii) Setting $k=1$ in (2.2) relations for lower records can be obtained as

$$
E\left(X_{L(r)}^{j}\right)=E\left(X_{L(r-1)}^{j}\right)-\frac{j}{a}\left\{Q E\left(\phi\left(X_{L(r)}\right) e^{a / h\left(x_{L(r)}\right)}\right)-E\left(\phi\left(X_{L(r)}\right)\right)\right\} .
$$

Remark 2.2. At $Q=0$ and $P=1$, (non-truncated case) relations (2.1) and (2.2) reduce, respectively, to

$$
E\left[X^{* j}(r, n, m, k)\right]-E\left[X^{* j}(r-1, n, m, k)\right]=-\frac{j}{a \gamma_{r}} E\left[\phi\left(X^{*}(r, n, m, k)\right)\right]
$$

and

$E\left[X^{* j}(r, n,-1, k)\right]-E\left[X^{* j}(r-1, n,-1, k)\right]=-\frac{j}{a k} E\left[\phi\left(X^{*}(r, n,-1, k)\right)\right]$.

The order statistics and lower record values cases are given from (2.5) and (2.6) as

$$
E\left(X_{n-r+1: n}^{j}\right)=E\left(X_{n-r+2: n}^{j}\right)-\frac{j}{a(n-r+1)} E\left(\phi\left(X_{n-r+1: n}\right)\right) .
$$

That is

$$
\left.E\left(X_{r: n}^{j}\right)=E\left(X_{r-1: n}^{j}\right)\right]+\frac{j}{a(r-1)} E\left(\phi\left(X_{r-1: n}\right)\right)
$$

and

$$
E\left(X_{L(r)}^{j}\right)=E\left(X_{L(r-1)}^{j}\right)-\frac{j}{a} E\left(\phi\left(X_{L(r)}\right)\right) .
$$

Similarly several recurrence relations based on Theorem 2.1 can be established with proper choice of $a$ and $h(x)$. 
TABLE 1. Examples Based on Theorem 2.1

\begin{tabular}{|c|c|c|c|}
\hline Distribution & $F(x)$ & $a$ & $h(x)$ \\
\hline Inverse Weibull & $\begin{array}{c}e^{-(\theta / x)^{p}} \\
0<x<\infty\end{array}$ & $\theta^{p}$ & $x^{p}$ \\
\hline Exponentiated Weibull & $\begin{array}{c}{\left[1-e^{-(\lambda x)^{p}}\right]^{\tau}} \\
0<x<\infty\end{array}$ & 1 & {$\left[-\ln \left\{1-e^{-(\lambda x)^{p}}\right\}^{\tau}\right]^{-1}$} \\
\hline Power function & $\begin{array}{c}(x / \lambda)^{p} \\
0<x<\lambda\end{array}$ & 1 & {$\left[-\ln (x / \lambda)^{p}\right]^{-1}$} \\
\hline Exponentiated Pareto & $\begin{array}{c}{\left[1-(1+x)^{-\lambda}\right]^{\theta}} \\
0<x<\infty\end{array}$ & 1 & {$\left[-\ln \left\{1-(1+x)^{-\lambda}\right\}^{\theta}\right]^{-1}$} \\
\hline Exponentiated gamma & $\begin{array}{c}{\left[1-e^{-x}(x+1)\right]^{\theta}} \\
0<x<\infty\end{array}$ & 1 & {$\left[-\ln \left\{1-e^{-x}(x+1)\right\}^{\theta}\right]^{-1}$} \\
\hline Generalized exponential & $\begin{array}{l}{\left[1-e^{-\lambda x}\right]^{\theta}} \\
0<x<\infty\end{array}$ & 1 & {$\left[-\ln \left(1-e^{-\lambda x}\right)^{\theta}\right]^{-1}$} \\
\hline Exponentiated log-logistic & $\begin{array}{l}{\left[\frac{(x / \sigma)^{\beta}}{1+(x / \sigma)^{\beta}}\right]^{\theta}} \\
0<x<\infty\end{array}$ & 1 & $\left.-\ln \left(\frac{(x / \sigma)^{\beta}}{1+(x / \sigma)^{\beta}}\right)^{\theta}\right]^{-1}$ \\
\hline Generalized inverse Weibull & $\begin{array}{l}e^{-\theta(\alpha / x)^{\beta}} \\
0<x<\infty\end{array}$ & $\theta$ & $(\alpha / x)^{-\beta}$ \\
\hline Extended type I generalized logistic & $\begin{array}{c}\left(\frac{\lambda}{\lambda+e^{-x}}\right)^{p} \\
-\infty<x<\infty\end{array}$ & 1 & {$\left[-\ln \left(\frac{\lambda}{\lambda+e^{-x}}\right)^{p}\right]^{-1}$} \\
\hline Logistic & $\begin{array}{c}{\left[1+e^{-x}\right]^{-1}} \\
-\infty<x<\infty\end{array}$ & 1 & {$\left[\ln \left(1+e^{-x}\right)\right]^{-1}$} \\
\hline Gumbel & $\begin{array}{c}e^{-e^{-x}} \\
-\infty<x<\infty\end{array}$ & 1 & $e^{x}$ \\
\hline
\end{tabular}

\section{Characterization}

THEOREM 3.1. Let $X$ be a non-negative random variable having an absolutely continuous distribution function $F(x)$ with $F(0)=0$ and $0<F(x)<1$ for all $x>0, m>-1$ then

$$
\begin{aligned}
& E\left[X^{* j}(r, n, m, k)\right]-E\left[X^{* j}(r-1, n, m, k)\right] \\
& =\frac{j}{a \gamma_{r}}\left\{Q E\left[\phi\left(X^{*}(r, n, m, k)\right) e^{a / h\left(X^{*}(r, n, m, k)\right)}\right]-E\left[\phi\left(X^{*}(r, n, m, k)\right)\right]\right\}
\end{aligned}
$$

if and only if

$$
F(x)=-Q_{2}+\frac{h^{2}(x)}{a h^{\prime}(x)} f(x), P_{1} \leq x \leq Q_{1} .
$$

Proof. The necessary part follows immediately from equation (2.1). On the other hand if the recurrence relation in equation (3.1) is satisfied, 
then on using equations (1.2) and (2.4), we have

$$
\begin{aligned}
& \frac{C_{r-1}}{(r-1) !} \int_{Q_{1}}^{P_{1}} x^{j}[F(x)]^{\gamma_{r}-1} f(x) g_{m}^{r-1}(F(x)) d x \\
& =\frac{(r-1) C_{r-1}}{\gamma_{r}(r-1) !} \int_{Q_{1}}^{P_{1}} x^{j}[F(x)]^{\gamma_{r}+m} f(x) g_{m}^{r-2}(F(x)) d x \\
& \quad+\frac{j Q C_{r-1}}{a \gamma_{r}(r-1) !} \int_{Q_{1}}^{P_{1}} \frac{x^{j-1} h^{2}(x) e^{a / h(x)}}{h^{\prime}(x)}[F(x)]^{\gamma_{r}-1} f(x) g_{m}^{r-1}(F(x)) d x \\
& \quad-\frac{j C_{r-1}}{a \gamma_{r}(r-1) !} \int_{Q_{1}}^{P_{1}} \frac{x^{j-1} h^{2}(x)}{h^{\prime}(x)}[F(x)]^{\gamma_{r}-1} f(x) g_{m}^{r-1}(F(x)) d x .
\end{aligned}
$$

Integrating the first integral on the right hand side of equation (3.2), by parts, we get

$$
\begin{aligned}
& \frac{C_{r-1}}{(r-1) !} \int_{Q_{1}}^{P_{1}} x^{j}[F(x)]^{\gamma_{r}-1} f(x) g_{m}^{r-1}(F(x)) d x \\
& =\frac{j C_{r-1}}{\gamma_{r}(r-1) !} \int_{Q_{1}}^{P_{1}} x^{j-1}[F(x)]^{\gamma_{r}} g_{m}^{r-1}(F(x)) d x \\
& \quad+\frac{C_{r-1}}{(r-1) !} \int_{Q_{1}}^{P_{1}} x^{j}[F(x)]^{\gamma_{r}-1} f(x) g_{m}^{r-1}(F(x)) d x \\
& \quad+\frac{j Q C_{r-1}}{a \gamma_{r}(r-1) !} \int_{Q_{1}}^{P_{1}} \frac{x^{j-1} h^{2}(x) e^{a / h(x)}}{h^{\prime}(x)}[F(x)]^{\gamma_{r}-1} f(x) g_{m}^{r-1}(F(x)) d x \\
& \quad-\frac{j C_{r-1}}{a \gamma_{r}(r-1) !} \int_{Q_{1}}^{P_{1}} \frac{x^{j-1} h^{2}(x)}{h^{\prime}(x)}[F(x)]^{\gamma_{r}-1} f(x) g_{m}^{r-1}(F(x)) d x
\end{aligned}
$$

which reduces to

$$
\begin{aligned}
& \frac{j C_{r-1}}{\gamma_{r}(r-1) !} \int_{Q_{1}}^{P_{1}} x^{j-1}[F(x)]^{\gamma_{r}-1} g_{m}^{r-1}(F(x))\left\{F(x)+Q_{2}-\frac{h^{2}(x)}{a h^{\prime}(x)} f(x)\right\} d x \\
& =0 .
\end{aligned}
$$

Now applying a generalization of the Müntz-Szász Theorem (Hwang and Lin, [10]) to equation (3.3), we get

$$
F(x)=-Q_{2}+\frac{h^{2}(x)}{a h^{\prime}(x)} f(x), P_{1} \leq x \leq Q_{1}
$$

which proves that $f(x)$ has the form as in equation (1.7). 
Now we shall use recurrence relation in (2.1), $Q=0$, to characterize the non-truncated general class of distribution by the following theorem.

Theorem 3.2. Let $X$ be a non-negative random variable having an absolutely continuous distribution function $F(x)$ with $F(0)=0$ and $0<F(x)<1$ for all $x>0$, then

$$
E\left[X^{* j}(r, n, m, k)\right]=E\left[X^{* j}(r-1, n, m, k)\right]-\frac{j}{a \gamma_{r}} E\left[\phi\left(X^{*}(r, n, m, k)\right)\right]
$$

if and only if

$$
F_{1}(x)=e^{-a / h(x)}, x \in(\alpha, \beta) .
$$

Proof. The necessary part follows immediately from equation (2.1). On the other hand if the recurrence relation in equation (3.4) is satisfied, then on using equations (1.2), we have

$$
\begin{aligned}
& \frac{C_{r-1}}{(r-1) !} \int_{\alpha}^{\beta} x^{j}\left[F_{1}(x)\right]^{\gamma_{r}-1} f_{1}(x) g_{m}^{r-1}\left(F_{1}(x)\right) d x \\
& =\frac{(r-1) C_{r-1}}{\gamma_{r}(r-1) !} \int_{\alpha}^{\beta} x^{j}\left[F_{1}(x)\right]^{\gamma_{r}+m} f_{1}(x) g_{m}^{r-2}\left(F_{1}(x)\right) d x \\
& \quad-\frac{j C_{r-1}}{a \gamma_{r}(r-1) !} \int_{\alpha}^{\beta} \frac{x^{j-1} h^{2}(x)}{h^{\prime}(x)}\left[F_{1}(x)\right]^{\gamma_{r}-1} f_{1}(x) g_{m}^{r-1}\left(F_{1}(x)\right) d x .
\end{aligned}
$$

Integrating the first integral on the right hand side of equation (3.5), by parts, we get

$$
\begin{aligned}
& \frac{C_{r-1}}{(r-1) !} \int_{\alpha}^{\beta} x^{j}\left[F_{1}(x)\right]^{\gamma_{r}-1} f_{1}(x) g_{m}^{r-1}\left(F_{1}(x)\right) d x \\
& =\frac{j C_{r-1}}{\gamma_{r}(r-1) !} \int_{\alpha}^{\beta} x^{j-1}\left[F_{1}(x)\right]^{\gamma_{r}} g_{m}^{r-1}\left(F_{1}(x)\right) d x \\
& \quad+\frac{C_{r-1}}{(r-1) !} \int_{\alpha}^{\beta} x^{j}\left[F_{1}(x)\right]^{\gamma_{r}-1} f_{1}(x) g_{m}^{r-1}\left(F_{1}(x)\right) d x \\
& \quad-\frac{j C_{r-1}}{a \gamma_{r}(r-1) !} \int_{\alpha}^{\beta} \frac{x^{j-1} h^{2}(x)}{h^{\prime}(x)}\left[F_{1}(x)\right]^{\gamma_{r}-1} f_{1}(x) g_{m}^{r-1}\left(F_{1}(x)\right) d x
\end{aligned}
$$

which reduces to

$$
\frac{j C_{r-1}}{\gamma_{r}(r-1) !} \int_{\alpha}^{\beta} x^{j-1}\left[F_{1}(x)\right]^{\gamma_{r}-1} g_{m}^{r-1}\left(F_{1}(x)\right)\left\{F_{1}(x)-\frac{h^{2}(x)}{a h^{\prime}(x)} f_{1}(x)\right\} d x=0 .
$$


Now applying a generalization of the Müntz-Szász Theorem (Hwang and Lin, [10]) to equation (3.3), we get

$$
\frac{f_{1}(x)}{F_{1}(x)}=\frac{a h^{\prime}(x)}{h^{2}(x)}
$$

which proves that

$$
F_{1}(x)=e^{-a / h(x)}, x \in(\alpha, \beta)
$$

\section{Conclusion}

This paper deals with the lower generalized order statistics within a class of doubly truncated distributions. Some recurrence relations for single moments are derived. Two theorems for characterizing the general form of distribution based on single moments of lower generalized order statistics are given. Special cases are also deduced.

\section{Acknowledgements}

The author is grateful to acknowledge with thanks to referees and Editor-in-Chief Journal of the Chungcheong Mathematical Society for carefully reading the paper and for helpful suggestions which greatly improved the paper.

\section{References}

[1] A. A. Ahmad, Moments of order statistics from doubly truncated continuous distributions and characterization, Statistics 35 (2001), 479-494.

[2] A. A. Ahmad, Recurrence relations for single and product moments of generalized order statistics from doubly truncated Burr type XII distribution, J. Egypt. Math. Soc. 15 (2007), 117-128.

[3] A. A. Ahmad and A. M. Fawzy, Recurrence relations for single moments of generalized order statistics from doubly truncated distributions, J. Statist. Plann. Inference 117 (2003) 241-249.

[4] M. Bieniek and D. Szynal, Characterizations of distributions via linearty of regression of generalized order statistics, Metrika 58 (2003) 259-271.

[5] M. Burkschat, E. Cramer, and U. Kamps, Dual generalized order statistics, Metron LXI (2003), 13-26.

[6] E. Camer and U. Kamps, Relations for expectations of functions of generalized order statistics, J. Statist. Plann. Inference 89 (2000), 79-89. 
[7] E. Camer, U. Kamps, and C. Keseling, Characterization via linear regression of ordered random variables: a unifying approach, Comm. Statist. Theory Methods 33 (2004), 2885-2911.

[8] Z. Govindarajulu, Characterization of double exponential using moments of order statistics, Comm. Statist. Theory Methods 30 (2001), 2355-2372.

[9] Z. Grudzien and D. Szynal, On characterizations of continuous distributions in terms of moments of order statistics when the sample size is random, J. Math. Sci. 92 (2001), 4017-4022.

[10] J. S. Hwang and G. D. Lin, On a generalized moments problem II Proc. Amer. Math. Soc. 91 (1984), 577-580.

[11] U. Kamps, A concept of Generalized Order Statistics, J. Statist. Plann. Inference 48 (1995), 1-23.

[12] U. Kamps, Characterizations of distributions by recurrence relations and identities for moments of order statistics. In: Balakrishnan, N. And C. Rao, C. R. eds., Hand book of statistics, 16, Order Statistics, Theory and Methods, Amsterdam: North Holand 16 (1998), 291-231.

[13] C. Keseling, Conditional distributions of generalized order statistics and some characterizations, Metrika 49 (1999), 27-40.

[14] A. H. Khan and A. M. Abouammoh, Characterizations of distributions by conditional expectation of order statistics, J. Appl. Statist. Sci. 9 (1999), 159-168.

[15] A. H. Khan, R. U. Khan, and M. Yaqub, Characterization of continuous distribution through conditional Expectation of functions of generalized order statistics, J. Appl. Probab. Statist. 1 (2006), 115-131.

[16] R. U. Khan, H. Athar, and Z. Anwer, Recurrence relations for single and product moments of generalised order statistics from doubly truncated Weibull distribution, Aligarh J. Statist. 27 (2007), 69-79.

[17] P. Pawlas and D. Szynal, Recurrence relations for single and product moments of lower generalized order statistics from the inverse Weibull distribution, Demonstratio Math. XXXIV (2001), 353-358.

[18] J. Wu and L. Y. Ouyang, On characterizing distributions by conditional expectations of functions of order statistics, Metrika 34 (1996), 135-147.

Department of Statistics, Amity Institute of Applied Sciences

Amity University

Noida-201 303, India

E-mail: devendrastats@gmail.com 